\title{
Autoavaliação de saúde, envolvimento social e fragilidade em idosos ambulatoriais
}

\author{
Self-rated health, social involvement and frailty in elderly outpatients
}

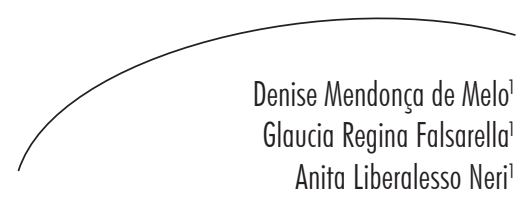

\section{Resumo}

Objetivos: Investigar relações entre autoavaliação global de saúde e de saúde referenciada a comparação social, fragilidade biológica e envolvimento social indicado por atividades avançadas de vida diária (AAVD) em idosos ambulatoriais. Métodos: 150 idosos (60 anos de idade e mais) sem déficits auditivos, visuais e cognitivos e com satisfatória comunicação verbal foram avaliados em suas características demográficas, autopercepção de saúde, critérios que integram as medidas de fragilidade de Fried et al.,22 e cinco domínios de AAVD. Resultados: A amostra com 96 mulheres $(\mathrm{m}=77,2$ anos $\pm 6,7)$ e 54 homens $(\mathrm{m}=76$ anos $\pm 8,5)$ foi estratificada em três grupos etários, com predominância de 70 a 79 anos. A maioria avaliou a própria saúde como de qualidade intermediária (50\%) ou boa $(31,8 \%)$. A categoria de AAVD mais frequente foi a social. Os homens se destacaram pelo desempenho significativamente maior de AAVD do domínio físico; 56\% dos idosos foram classificados como frágeis; $41,2 \%$ como pré-frágeis, destacando-se as mulheres e aqueles com 70 anos e mais. Os frágeis apresentaram piores autoavaliações de saúde e de saúde comparada a de outros da mesma idade e pior desempenho de AAVD, com destaque para as mulheres e para aqueles com 80 anos e mais. Conclusões: A autoavaliação de saúde positiva e a manutenção do envolvimento social integram condições para amenizar o impacto da possível diminuição do desempenho de atividades complexas de vida diária em idosos frágeis ambulatoriais.

\section{Abstract}

Objectives: To investigate relationships between global self-rated health and health referred to social comparison, biological frailty and social involvement indicated by advanced activities of daily living (AADL) in elderly outpatients. Methods: 150 participants (60 years or more) without auditory, visual and cognitive deficits and satisfactory verbal communication were evaluated on their demographic characteristics, self-rated health, the criteria of frailty of Fried et al. ${ }^{22}$ and five AADL domains. Results: The sample consisted of 96 women $(\mathrm{m}=77.2$ years $\underline{+6.7)}$ ) and $54 \mathrm{men}(\mathrm{m}=76$ years $\underline{+8.5})$, stratified into three age groups, with prevalence of $70-79$ years. Most rated their health as intermediate

Palavras-chave: Saúde. Participação Social. Funcionalidade. Fragilidade. Idoso.
Key words: Health. Social Participation. Functionality. Frailty. Elderly.

\footnotetext{
Programa de Pós-graduação em Gerontologia, Faculdade de Ciências Médicas. Universidade Estadual de Campinas. Campinas, SP, Brasil. 
quality $(50 \%)$ or good $(31.8 \%)$. The most frequent AADL category was social. Men stood out for significantly higher performance in physical domain AADL; $56 \%$ of the elderly were classified as frail; $41.2 \%$ as pre-frail, especially for women and those over 70 years old. The frail elderly self-assessments showed worse health and health compared to others of the same age and worse AADL performance, especially for women and for those aged 80 years and more. Conclusions: The positive self-assessment of health plus the maintenance of positive social involvement are conditions to soften the impact of possible decrease in performance of complex activities of daily living in frail elderly outpatients.

\section{INTRODUÇÃO}

Considerada como o principal indicador de qualidade de vida percebida, a autoavaliação de saúde é um conceito corrente na pesquisa epidemiológica sobre saúde do idoso. É o indicador mais comumente utilizado para investigar o bem-estar físico e mental. ${ }^{1,2}$ É acessada por meio de escalas como o Health Perceptions Questionnaire (Form II), ${ }^{3}$ dividido em 36 itens estruturados ou, mais comumente, por item único, dicotômico ou escalar, que solicita que o respondente classifique a qualidade de sua saúde. O julgamento subjetivo dos idosos sobre suas condições de saúde tem bases pessoais, lastreadas por informações oriundas de profissionais de saúde, por experiências fisiológicas, pela observação do próprio comportamento e pela observação do comportamento de outrem.

Ao comparar sua saúde com a de outros da mesma idade que se encontram em pior condição, os idosos podem favorecer a autoestima. Os processos de comparação social desempenham funções adaptativas. Operam por meio de comparações laterais, descendentes e ascendentes com outras pessoas. ${ }^{4} \mathrm{~A}$ comparação social é adotada pelos idosos com o objetivo de validar e calibrar as avaliações feitas pelo self em relação a características de personalidade, comportamentos, capacidades, realizações atuais e passadas e opiniões. ${ }^{5}$ Esse conjunto de dados é confrontado com expectativas e valores que, por sua vez, são baseados em normas socioculturais.

A autopercepção de saúde prediz de forma robusta a morbidade, a incapacidade, a depressão, a inatividade e a mortalidade em idosos, ${ }^{2,6-8}$ motivo pelo qual é considerada tão importante para prever o curso da saúde ao longo do tempo 9 quanto dados objetivos sobre saúde. Os idosos geralmente avaliam sua saúde de forma positiva ou muito positiva. ${ }^{1,10}$

O Estudo Fibra Campinas investigou a autopercepção de saúde em 689 indivíduos com 65 anos de idade e mais, residentes na comunidade, e verificou que $71,3 \%$ da amostra consideraram sua saúde como boa e muito boa; $24,3 \%$ como regular; e $4,3 \%$ como ruim e muito ruim. ${ }^{10}$ Rodrigues et al. ${ }^{11}$ analisaram as características de 4.003 idosos brasileiros das regiões Nordeste e Sul que faziam uso de serviços ambulatoriais. Na Região Sul, 60,7\% dos idosos autoavaliaram sua saúde como regular/ ruim e 39,3\% como excelente, boa e muito boa. $\mathrm{Na}$ Região Nordeste, os valores foram 67,9\% e $32,1 \%$, respectivamente. A Pesquisa Nacional por Amostra de Domicílios (PNAD) avaliou, entre os anos de 1998 a 2008, as tendências de saúde da população idosa brasileira (105.254 idosos), e constatou aumento gradativo da boa autoavaliação da saúde ao longo de dez anos, passando de 39,3\% para $43,5 \%$, e posteriormente 45,0\% para os anos de 1998, 2003 e $2008 .{ }^{1}$

A saúde percebida avaliada positivamente relaciona-se com maior sobrevida, percepção de controle e uso de estratégias de manutenção da saúde e do bem-estar, além de maior envolvimento em atividades complexas da vida diária. ${ }^{12} \mathrm{~A}$ autopercepção negativa de saúde entre os idosos guarda forte associação com gênero feminino, baixa renda, comorbidades, depressão, incapacidades, inatividade física, acesso e uso deficitário de serviços de saúde ${ }^{7,13}$ e isolamento 
social; ${ }^{14}$ insatisfação com os relacionamentos pessoais, menor frequência a clubes e associações, sintomas depressivos, queixa de insônia, maior número de medicamentos prescritos, dificuldade de acesso aos serviços de saúde, maior número de consultas médicas nos últimos 12 meses e mais internações hospitalares no período; ${ }^{6}$ baixo status de saúde e incapacidades funcionais, ${ }^{2}$ inatividade e isolamento social. ${ }^{12}$

A interação entre boas condições de saúde, funcionalidade, adequação ambiental e presença de recursos pessoais resulta em maior envolvimento em atividades sociais. ${ }^{15}$ Estas exigem competência comportamental física e cognitiva suficientes para o manejo do ambiente físico e social mais amplo, motivo pelo qual são reconhecidas como atividades avançadas de vida diária (AAVD). ${ }^{16,17} \mathrm{O}$ desempenho de AAVD como trabalhar e fazer parte de associações cívicas, recreativas e culturais permite que o idoso seja considerado participativo, envolvido, ativo e produtivo. A diminuição na frequência de AAVD de natureza social sinaliza perda de motivação para contatos sociais, problemas de mobilidade e inadequação ambiental. Consideradas como eventos sentinela do declínio funcional, as perdas em AAVD sociais precedem dificuldades em atividades instrumentais de vida diária (AIVD) e em atividades básicas de vida diária (ABVD). ${ }^{13}$

A fragilidade associa-se à incapacidade funcional e à restrição de atividades entre os idosos. ${ }^{18} \mathrm{Al}$ Snih et al. ${ }^{19}$ examinaram a associação entre fragilidade e incapacidade em 1.645 idosos por dez anos. Identificaram que, inicialmente, $50 \%$ deles não eram frágeis, $45,7 \%$ eram préfrágeis e 4,3\% eram frágeis. Em dez anos, o risco para incapacidade nos pré-frágeis e frágeis, em comparação aos robustos, foi de 1,32 e 2,42, respectivamente. Kono et al. ${ }^{20}$ estudaram 107 idosos frágeis ambulatoriais quanto à frequência de saídas de casa. O grupo que na avaliação em linha de base disse que saía de casa mais de quatro vezes por semana preservou suas atividades de vida diária (AVD) por um período maior do que 20 meses, enquanto o grupo que saía entre uma a três vezes manteve suas AVDs por, no máximo, nove meses.
Idosos ambulatoriais reúnem características peculiares, como maior morbidade, fragilidade, incapacidade e depressão, além de baixo envolvimento social. ${ }^{21}$ Essas particularidades inspiram atenção especial. Em termos clínicos, é relevante compreender as relações entre a autoavaliação de saúde, a fragilidade e o envolvimento social. No entanto, poucos estudos brasileiros analisaram a saúde percebida em relação com envolvimento social e fragilidade em idosos ambulatoriais.

O objetivo desta pesquisa foi investigar relações entre autoavaliação global de saúde, autoavaliação de saúde referenciada à comparação social, fragilidade biológica e envolvimento social indicado por atividades avançadas de vida diária em amostra de mulheres e homens idosos com 60 anos de idade e mais atendidos em ambulatório de Geriatria.

\section{METODOLOGIA}

\section{Participantes}

Participaram deste estudo 150 idosos com 60 anos de idade e mais, atendidos no Ambulatório de Geriatria do Hospital de Clínicas da Faculdade de Ciências Médicas da Universidade Estadual de Campinas entre outubro de 2005 e setembro de 2007. Nesse período, o Ambulatório de Geriatria realizou 1.020 consultas médicas. Os critérios de elegibilidade foram: a) ter 60 anos ou mais; b) aceitar participar da pesquisa; e c) assinar um Termo de Consentimento Livre e Esclarecido. Os critérios de exclusão foram: a) déficit auditivo ou visual não compensados por aparelhos; b) comunicação verbal comprometida; e c) déficit cognitivo grave sugestivo de demência informado no prontuário médico do idoso.

Variáveis e instrumentos

1. Identificação: foram feitas perguntas sobre o nome do idoso, o nome do acompanhante e a natureza de seu parentesco com o idoso, e foi registrado o número de cadastro no hospital de cada um deles. 
2. Características demográficas: a variável "gênero" incluiu as alternativas "masculino" e "feminino". A idade foi derivada da data de nascimento informada pelos idosos. Os dados foram agrupados em três faixas: $<70,70$ a 79 e $>80$.

3. Autoavaliação de saúde: foram utilizadas duas questões com possibilidades de resposta "ruim", "mais ou menos" ou "boa" para ambas. A primeira dizia respeito à avaliação global da saúde (“Como é a sua saúde de modo geral?”) e a segunda solicitava que os idosos considerassem como critério a comparação social com outros da mesma idade ("Como é a sua saúde, em comparação com a de outras pessoas da sua idade?”).

4. Fragilidade: esta condição foi avaliada de acordo com a definição operacional de Fried et al., ${ }^{22}$ que consideram cinco critérios individuais e a combinação deles para a categorização dos idosos como frágeis (os que pontuam para três ou mais critérios), pré-frágeis (os que pontuam para um ou dois critérios) e não frágeis (os que não pontuam para nenhum dos critérios).

\subsection{Perda de peso não intencional no ano} anterior: foi utilizada a pergunta "O senhor perdeu peso no último ano?”. Em caso de resposta afirmativa, era feita uma pergunta sobre a quantidade. Pontuaram para perda de peso os idosos que relataram ter perdido $4,5 \mathrm{~kg}$ ou mais ou um valor igual ou maior do que $5 \%$ do peso corporal.

4.2. Fadiga: foram utilizadas duas questões da escala CES-D (The Center for Epidemiologic Studies - Depression) ${ }^{23,24}$ com referência aos últimos sete dias: "Sentiu que teve que fazer esforço para dar conta das suas tarefas de todo dia?" e "Sentiu que não conseguiu levar adiante as suas coisas?", ambas com opções de resposta "nunca/ raramente", "poucas vezes", "na maioria das vezes" e "sempre". Os idosos que respondiam "sempre" ou "quase sempre" a qualquer uma das duas perguntas pontuavam para fragilidade.

4.3. Nível de atividade física: perguntavase aos idosos se praticavam exercícios físicos regularmente, com opções de resposta "sim" ou "não". Para os que respondiam sim, perguntavase pelos tipos de exercícios físicos praticados, quantas vezes por semana e por quanto tempo por dia. Foram considerados ativos aqueles que acumularam pelo menos 120 minutos semanais em exercícios físicos e esportes ativos de intensidade vigorosa ( $>6 \mathrm{MET}$ ) ou aqueles que acumularam mais de 150 minutos semanais em exercícios físicos e esportes ativos de intensidade moderada (de $\geq 3$ MET a $\leq 6$ MET). ${ }^{25,26}$

4.4. Força de preensão manual: foi medida com dinamômetro analógico, marca Crown, modelo manual $\left(\right.$ Cielab $^{\circledR}$ Equipamentos para Laboratórios Científicos Ltda., Campinas-SP, Brasil), com capacidade de 50 quilogramasforça (kgf), ajustável, em três tentativas, com intervalo de um minuto entre elas. A aferição era feita com o idoso em posição ortostática, com o dinamômetro na mão dominante, com os dois cotovelos estendidos e os dois punhos em posição neutra. Para cada idoso, foi registrado o resultado do melhor desempenho. Pontuaram para baixa força de preensão manual os idosos cuja medida em $\mathrm{kgf}$ situou-se abaixo do $1^{\circ}$ quintil da distribuição, com ajuste por sexo e por índice de massa corporal (IMC). ${ }^{22}$

4.5. Velocidade da marcha: os idosos foram convidados a percorrer duas vezes um trajeto de quatro metros delimitado com fita adesiva, em linha reta, em passo usual, sendo permitido o uso de bengala ou andador. Foi considerado o tempo em segundos gasto no segundo trecho, com ajustamento por sexo e por estatura. Pontuaram para lentidão os idosos cujo tempo em segundos foi superior ao percentil $80 \mathrm{da}$ distribuição. ${ }^{22}$

5. Inventário de atividades avançadas de vida diária (AAVD): com base em Reuben et al. ${ }^{16}$ e Baltes et al., ${ }^{17}$ foi construído um inventário com 20 itens sobre atividades avançadas de vida diária de natureza física (passear/caminhar; jardinagem; artesanato; exercícios físicos; dirigir); social (ir a reuniões sociais; festas e bailes; fazer visitas; receber visitas; trabalho remunerado e voluntário; frequentar a igreja para cultos; frequentar grupos na igreja; participar de diretorias e conselhos); físico-social (cuidar de netos e bisnetos e cuidar 
de adulto ou idoso doente); intelectual (frequentar cinema, teatro e concertos; passatempos intelectuais, como ler e organizar coleções; fazer cursos ou participar de grupos de estudo e realizar atividades artísticas); e intelectual-social (assistir TV, ouvir rádio e apreciar música). Em cada item, havia três opções de resposta: "sempre", "de vez em quando", "nunca".

\section{Coleta de dados}

Os idosos foram avaliados individualmente por membros de uma equipe multiprofissional, em salas reservadas localizadas no Ambulatório de Geriatria do Hospital de Clínicas da Faculdade de Ciências Médicas da Unicamp, em sessão única com 90 a 120 minutos de duração. Eram convidados a participar por ordem de chegada. Os dados levantados faziam parte de um protocolo que incluía dados demográficos; medidas clínicas, antropométricas e de força; medidas de autorrelato sobre condições de saúde física e bucal, fragilidade, desempenho de AVD, rastreio cognitivo, mobilidade e flexibilidade, hábitos de vida, satisfação com a alimentação e com sua funcionalidade, quedas, sintomas depressivos e bem-estar subjetivo.

\section{Análise de dados}

As análises estatísticas foram feitas por meio dos pacotes SPSS ${ }^{\circledR}$, versão 15.1, e do SAS ${ }^{\circledR}$, versão 8.02. A partir da aplicação do teste de normalidade Kolmogorov-Smirnov, verificou-se que as distribuições não eram normais, motivo pelo qual foram adotados testes não paramétricos para comparar as frequências das variáveis nominais (qui-quadrado e Exato de Fisher) e para comparar os valores das variáveis ordinais (Mann-Whitney e KruskalWallis). Foi feita análise de conglomerados, pelo método de partição, para identificar modelos de agrupamento para as variáveis: gênero, idade, critérios de fragilidade, autoavaliação de saúde, saúde comparada e AAVD. O valor de $\mathrm{p} \leq 0,05$ foi assumido como indicador de significância estatística para todas as análises.

\section{Considerações éticas}

Antes da sessão de coleta de dados, ainda na sala de espera, eram feitos os convites individuais aos idosos. Eram explicados os objetivos, o caráter voluntário da participação, a natureza das medidas, a duração aproximada da sessão, o direito ao sigilo dos dados individuais, a ausência de riscos à integridade física e psicológica, o caráter gratuito da participação e a ausência de remuneração. Os idosos e seus acompanhantes recebiam garantia de que a participação não comprometeria a realização da consulta médica no mesmo dia. Foi facultado aos idosos se fazerem acompanhar por uma pessoa à sua escolha na sessão de coleta de dados, mas os acompanhantes não participaram das entrevistas. Todos os idosos assinaram um Termo de Consentimento Livre e Esclarecido. O projeto foi aprovado pelo Comitê de Ética em Pesquisa da Faculdade de Ciências Médicas da Universidade Estadual de Campinas, sob o n ${ }^{\circ} 240 / 2003$.

\section{RESULTADOS}

A amostra compreendia 96 mulheres e 54 homens, distribuídos por três grupos de idade (<70 anos, 70 a 80 e $>80$ anos), com predominância daqueles de idade intermediária $(43,3 \%)$ e avançada (34\%). A média de idade foi $77,2 \pm 6,7$ anos entre as mulheres e $76 \pm 8,5$ anos entre os homens. A maioria avaliou a própria saúde como de qualidade intermediária (50\%) ou boa $(31,8 \%)$, sem diferenças estatisticamente significativas entre os grupos de idade e gênero. Proporção significativamente maior de homens do que de mulheres julgou que sua saúde era de qualidade intermediária ou boa, quando comparada com a de outros da mesma idade. Houve significativamente mais mulheres do que homens e mais idosos de 80 anos e mais e de 70 a 79 anos que pontuaram para baixa força de preensão palmar e para lentidão da marcha. Houve um percentual significativamente maior de idosos mais velhos que pontuou para perda de peso (tabela 1). 
Tabela 1. Autoavaliação da saúde atual, saúde comparada com outros da mesma idade e indicadores de fragilidade, conforme gênero e idade. Campinas-SP, 2005-2007.

\begin{tabular}{|c|c|c|c|c|c|c|c|c|}
\hline & & \multicolumn{3}{|c|}{ Gênero } & \multicolumn{4}{|c|}{ Idade } \\
\hline & & $\begin{array}{c}\text { Feminino } \\
\mathrm{n}(\%)\end{array}$ & $\begin{array}{c}\text { Masculino } \\
\mathrm{n}(\%)\end{array}$ & valor-p* & $\begin{array}{l}<70 \\
\mathrm{n}(\%)\end{array}$ & $\begin{array}{l}70-79 \\
\mathrm{n}(\%)\end{array}$ & $\begin{array}{l}>80 \\
\mathrm{n}(\%)\end{array}$ & valor-p \\
\hline \multirow[t]{3}{*}{ Idade } & $<70$ & $27(8,1)$ & $7(12,9)$ & 0,061 & & & & \\
\hline & 70-79 & $36(37,5)$ & $29(53,8)$ & & & & & \\
\hline & $>80$ & $33(34,4)$ & $18(33,3)$ & & & & & \\
\hline \multirow{3}{*}{$\begin{array}{l}\text { Autoav. } \\
\text { saúde }\end{array}$} & Ruim & $22(23,4)$ & $5(9,2)$ & 0,074 & $8(25,6)$ & $9(13,9)$ & $5(10,3)$ & 0,177 \\
\hline & Interm. & $42(44,7)$ & $32(59,3)$ & & $21(61,7)$ & $22(33,8)$ & $16(32,5)$ & \\
\hline & Boa & $47(31,9)$ & $17(31,5)$ & & $5(14.7)$ & $34(52,3)$ & $28(57,2)$ & \\
\hline Saúde & Ruim & $17(\mathbf{1 8 , 1 )}$ & $3(5,5)$ & 0,027 & $6(17,6)$ & $9(13,9)$ & $5(10,2)$ & 0,794 \\
\hline \multirow[t]{2}{*}{ comp. } & Interm. & $35(37,2)$ & $16(29,5)$ & & $13(38,3)$ & $22(33,8)$ & $16(32,6)$ & \\
\hline & Boa & $42(44,7)$ & $35(65,0)$ & & $15(44,1)$ & $34(52,3)$ & $28(57,2)$ & \\
\hline Níveis & Não & $2(2,1)$ & $2(3,7)$ & 0,085 & $3(8,8)$ & $0(0)$ & $1(2,0)$ & 0,001 \\
\hline \multirow[t]{2}{*}{ fragil. } & Pré & $34(35,4)$ & $28(51,9)$ & & $21(61,8)$ & $24(37,0)$ & $17(33,3)$ & \\
\hline & Frágeis & $60(62,5)$ & $24(44,4)$ & & $10(29,4)$ & $41(63,0)$ & $33(64,7)$ & \\
\hline Perda & $\operatorname{Sim}$ & $40(45,0)$ & $25(43,1)$ & 0,719 & $6(18,2)$ & $37(61,7)$ & $22(45,8)$ & $<0,001$ \\
\hline peso & Não & $49(55,0)$ & $27(51,9)$ & & $27(81,2)$ & $23(38,3)$ & $26(54,1)$ & \\
\hline \multirow[t]{2}{*}{ Fadiga } & Sim & $29(31,2)$ & $11(20,4)$ & 0,156 & $11(32,3)$ & $18(28,1)$ & $11(22,4)$ & 0,594 \\
\hline & Não & $64(68,2)$ & $43(79,6)$ & & $23(67,5)$ & $46(71,9)$ & $38(77,5)$ & \\
\hline Baixa f. & $\operatorname{Sim}$ & $62(64,5)$ & $26(48,1)$ & 0,049 & $13(38,2)$ & $41(63,0)$ & $34(66,7)$ & 0,021 \\
\hline preens. & Não & $34(35,4)$ & $28(51,8)$ & & $21(61,7)$ & $24(37,0)$ & $17(33,3)$ & \\
\hline Lent. & $\operatorname{Sim}$ & $61(63,5)$ & $21(39,0)$ & 0,004 & $10(29,4)$ & $41(63,0)$ & $31(60,8)$ & 0,003 \\
\hline marcha & Não & $35(36,4)$ & $33(61,1)$ & & $24(\mathbf{7 0 , 6 )}$ & $24(37,0)$ & $20(39,2)$ & \\
\hline Inativ. & $\operatorname{Sim}$ & $74(77,9)$ & $46(85,2)$ & 0,280 & $28(82,3)$ & $51(78,5)$ & $41(82,0)$ & 0,853 \\
\hline física & Não & $21(22,1)$ & $8(14,8)$ & & $6(17,6)$ & $14(21,5)$ & $9(18,0)$ & \\
\hline
\end{tabular}

*Teste qui-quadrado; diferença significativa se $\mathrm{p} \leq 0,005$.

As AAVDs desempenhadas por mais idosos foram "assistir televisão" (79\%), "cuidardojardim" $(44,30 \%)$ e "receber visitas" (42,95\%). "Frequentar igreja ou templo para funções religiosas" apareceu em quarto lugar (42,28\%). Veio em seguida um conjunto de atividades variadas, com frequências entre 23,50\% (passatempos intelectuais) e
$14,10 \%$ (fazer visitas). Entre as AAVDs nunca desempenhadas pelos idosos, foram observadas "ir ao cinema, teatro ou concerto", "participar de diretoria", "cuidar de doentes", "dirigir", "fazer trabalho voluntário e remunerado", "frequentar cursos", "desempenhar atividades artísticas" e "participar de reuniões, festas e bailes" (figura 1). 


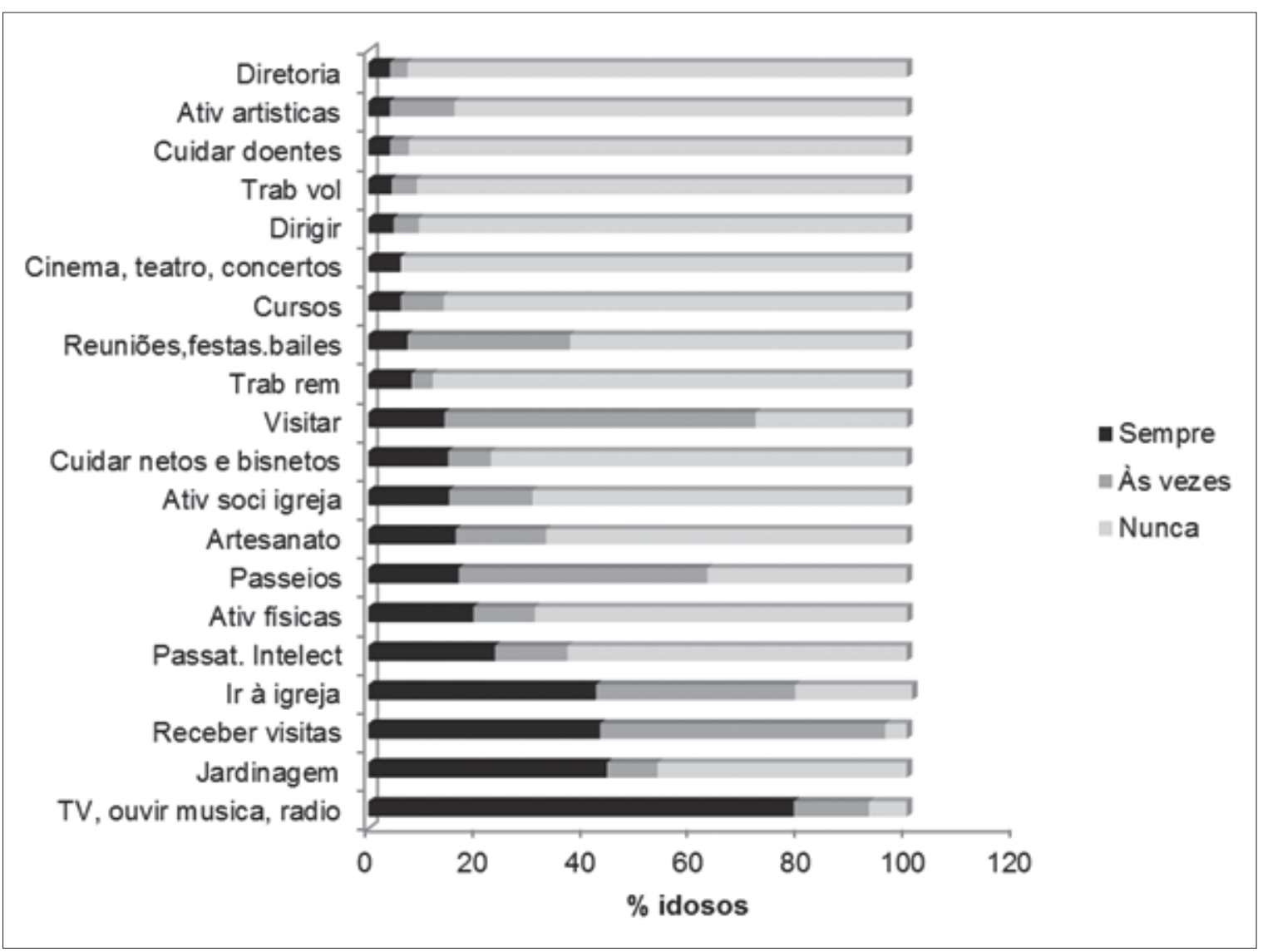

Figura 1. Frequência de AAVD, indicativas de envolvimento social. Campinas-SP, 2005-2007.

Dentre os idosos do estudo, $27 \%$ relataram fazer exercícios físicos com regularidade. Entre eles, $51,2 \%$ o faziam mais de quatro vezes por semana; $19,5 \%$, três ou quatro vezes na semana; $14,6 \%$, duas ou três vezes e $14,6 \%$, uma ou duas vezes por semana. Caminhada foi a atividade preponderante $(71 \%)$. Conforme os critérios do American College of Sports Medicine, ${ }^{27}$ apenas 19,46\% dos idosos se classificaram como ativos.

A categoria de AAVD mais frequente foi a social para os dois gêneros e para os três grupos de idade pesquisados. Os homens se destacaram pelo desempenho significativamente maior de AAVD do domínio físico (figura 1).

Cinquenta e seis por cento dos idosos foram classificados como frágeis, $41,2 \%$ como préfrágeis e 2,6\% como não frágeis. As mulheres e os idosos de 70 anos e mais pontuaram para maior número de critérios de fragilidade. Nas AAVDs físicas, a média dos homens superou a das mulheres de forma estatisticamente significativa (tabela 2). 
Tabela 2. Comparação entre os grupos de gênero e de idade com relação ao desempenho nas categorias de AAVD e ao número de critérios de fragilidade. Campinas-SP, 2005-2007.

\begin{tabular}{|c|c|c|c|c|c|c|c|}
\hline & \multicolumn{3}{|c|}{ Gênero } & \multicolumn{4}{|c|}{ Idade } \\
\hline & $\begin{array}{l}\text { Feminino } \\
\text { m (dp) }\end{array}$ & $\begin{array}{l}\text { Masculino } \\
\text { m (dp) }\end{array}$ & valor-p* & $\begin{array}{c}<70 \\
\mathrm{~m}(\mathrm{dp})\end{array}$ & $\begin{array}{l}\text { 70-79 } \\
\text { m (dp) }\end{array}$ & $\begin{array}{c}>80 \\
\mathrm{~m}(\mathrm{dp})\end{array}$ & valor-p ${ }^{* *}$ \\
\hline Idade & $76,0(8,5)$ & $77,2(6,7)$ & 0,348 & & & & \\
\hline AAVD & & & & & & & \\
\hline Físicas & $1,7(1,2)$ & $2,2(1,3)$ & 0,045 & $2,0(1,2)$ & $2,0(2,0)$ & $1,6(1,2)$ & 0,087 \\
\hline Sociais & $3,5(1,6)$ & $3,6(1,5)$ & 0,836 & $3,9(1,9)$ & $3,5(3,5)$ & $3,5(1,6)$ & 0,588 \\
\hline Físico-sociais & $0,2(0,5)$ & $0,4(0,5)$ & 0,225 & $0,3(1,5)$ & $0,3(0,4)$ & $0,2(0,4)$ & 0,283 \\
\hline Intelectuais & $0,5(0,7)$ & $0,7(0,7)$ & 0,076 & $0,4(0,6)$ & $0,7(0,7)$ & $0,5(0,6)$ & 0,073 \\
\hline Intelectuais-sociais & $0,9(0,3)$ & $0,9(0,2)$ & 0,264 & $0,9(0,3)$ & $0,9(0,9)$ & $0,9(0,3)$ & 0,653 \\
\hline $\begin{array}{l}\text { Número de critérios } \\
\text { de fragilidade }\end{array}$ & $2,8(1,1)$ & $2,4(1,1)$ & 0,042 & $2,0(1,0)$ & $2,9(1,0)$ & $2,7(1,0)$ & $<0,001$ \\
\hline
\end{tabular}

*Teste U de Mann Whitney; diferença significativa se <0,05.

**Teste Kruskal- Wallis; diferença significativa se $<0,05$.

A maioria avaliou a saúde como de qualidade intermediária, inclusive quando aplicado o critério à comparação social. Não foram observadas diferenças significativas entre as autoavaliações de saúde conforme os níveis de fragilidade, mas um percentual significativamente mais alto de idosos não frágeis e pré-frágeis considerou sua saúde como melhor do que a de outros da mesma idade, e um percentual mais alto de frágeis pontuou pior e igual. Houve mais idosos com fadiga e com lentidão da marcha que avaliaram negativamente a própria saúde (tabela 3).

Tabela 3. Autoavaliação de saúde atual e saúde comparada com outros da mesma idade, conforme os indicadores de fragilidade. Campinas-SP, 2005-2007.

\begin{tabular}{|c|c|c|c|c|c|c|c|c|c|}
\hline & & \multicolumn{4}{|c|}{ Autoavaliação da saúde } & \multicolumn{4}{|c|}{ Saúde comparada com outros da mesma idade } \\
\hline & & Ruim & Interm. & Boa & valor-p* & Ruim & Interm. & Boa & valor-p* \\
\hline Níveis de & Não + Pré & $8(12,5)$ & $34(53,1)$ & $22(34,4)$ & 0,286 & $6(9,4)$ & $17(26,5)$ & $41(64,0)$ & 0,037 \\
\hline fragilidade & Frágeis & $19(22,6)$ & $40(47,7)$ & $25(30,0)$ & & $14(16,7)$ & $34(40,5)$ & $36(42,9)$ & \\
\hline \multirow[t]{2}{*}{ Perda de peso } & Sim & $14(21,5)$ & $34(52,3)$ & $17(26,1)$ & 0,595 & $10(15,4)$ & $23(35,4)$ & $32(49,2)$ & 0,500 \\
\hline & Não & $13(17,5)$ & $36(48,5)$ & $25(33,8)$ & & $7(9,4)$ & $25(33,8)$ & $42(56,7)$ & \\
\hline \multirow[t]{2}{*}{ Fadiga } & $\operatorname{Sim}$ & $14(35,0)$ & $16(40,0)$ & $10(25,0)$ & 0,006 & $10(25,0)$ & $19(47,5)$ & $11(27,5)$ & $<0,001$ \\
\hline & Não & $13(12,1)$ & $58(54,2)$ & $36(33,6)$ & & $10(9,3)$ & $32(29,9)$ & $65(60,7)$ & \\
\hline Baixa força de & Sim & $18(20,7)$ & $41(47,1)$ & $28(32,2)$ & 0,591 & $11(12,7)$ & $29(33,4)$ & $47(54,0)$ & 0,836 \\
\hline preensão & Não & $9(14,8)$ & $33(54,1)$ & $19(31,1)$ & & $9(14,7)$ & $22(36,0)$ & $30(49,2)$ & \\
\hline \multirow[t]{2}{*}{ Lentidão da marcha } & $\operatorname{Sim}$ & $21(26,2)$ & $34(42,5)$ & $25(31,2)$ & 0,018 & $16(20,0)$ & $31(38,7)$ & $33(41,2)$ & 0,018 \\
\hline & Não & $6(8,9)$ & $40(58,9)$ & $22(32,2)$ & & $4(5,8)$ & $20(29,4)$ & $44(64,7)$ & \\
\hline Inatividade & $\operatorname{Sim}$ & $22(18,5)$ & $63(53,0)$ & $34(28,5)$ & 0,222 & $14(11,7)$ & $47(39,5)$ & $58(48,8)$ & 0,029 \\
\hline física & Não & $5(17,2)$ & $11(38,0)$ & $13(45,0)$ & & $6(20,7)$ & $4(13,8)$ & $19(65,5)$ & \\
\hline
\end{tabular}

*Teste Kruskal-Wallis; diferença significativa se $<0,05$. 
$\mathrm{Na}$ análise de conglomerados, o modelo que se mostrou mais satisfatório na explicação de variância dos dados foi o de quatro elementos, com a seguinte configuração:

- Conglomerado 1: predominantemente formado por mulheres, com 80 anos e mais, fisicamente inativos, com baixa força de preensão e baixa velocidade de marcha, saúde percebida como de qualidade intermediária, saúde avaliada como melhor do que a de outros da mesma idade, baixo número de AAVD nos domínios físico, social e físico-social e mais AAVD nos domínios intelectual e intelectual-social.

- Conglomerado 2: predominantemente formado por mulheres, com menos de 80 anos, baixa força de preensão, melhor saúde comparada, menor número de AAVD no domínio físico-social e maior número de AAVD nos domínios físico, social, intelectual e intelectual-social.

- Conglomerado 3: predominantemente formado por mulheres, com 70 a 79 anos, fisicamente inativas, com perda de peso maior que o critério, baixa força de preensão, baixa velocidade da marcha e fadiga, saúde percebida como negativa e pior do que a de outros da mesma idade, menor número de AAVD nos domínios físico, social, físico-social, intelectual e intelectual-social.

- Conglomerado 4: predominantemente formado por homens, com menos de 80 anos, fisicamente inativos, que haviam perdido peso acima do critério, que avaliaram sua saúde como de qualidade intermediária e de melhor qualidade do que a de outros da mesma idade, menor número de AAVD nos domínios social e intelectual, e maior número de AAVD nos domínios físico, físico-social e intelectual-social.

As variáveis que mais contribuíram para explicar os resultados foram: avaliação de saúde referenciada à comparação social $\left(\mathrm{R}^{2}=\right.$ $0,469)$; AAVD físicas $\left(\mathrm{R}^{2}=0,418\right)$; AAVD físicosociais $\left(R^{2}=0,400\right)$; AAVD sociais $\left(R^{2}=0,302\right)$ e inatividade física $\left(R^{2}=0,269\right)$. Os dados em detalhes podem ser apreciados na tabela 4 . 
Tabela 4. Conglomerados com os percentuais ou as médias de idosos das distribuições, conforme as variáveis: gênero, idade, autoavaliação de saúde, saúde comparada com outros da mesma idade, fragilidade e AAVD. Campinas-SP, 2005-2007.

\begin{tabular}{|c|c|c|c|c|c|c|c|c|c|c|}
\hline Conglomerados & & $\mathrm{n}$ & 1 & & 2 & & 3 & & 4 & valor-p ${ }^{*}$ \\
\hline \multirow[t]{2}{*}{ Gênero } & Feminino & 54 & 53,2 & & 81,0 & & 93,3 & & 48,6 & $<0,001$ \\
\hline & Masculino & 96 & 46,8 & & 19,0 & & 6,7 & & 51,4 & \\
\hline \multirow[t]{3}{*}{ Idade } & $<70$ & 34 & 9,7 & & 38,1 & & 20,0 & & 37,8 & $<0,001$ \\
\hline & $70-79$ & 65 & 38,1 & & 38,1 & & 50,0 & & 48,6 & \\
\hline & $>80$ & 51 & 51,6 & & 23,8 & & 30,0 & & 13,5 & \\
\hline Autoavaliação & Ruim & 27 & 11,6 & & 0 & & 56,6 & & 8,1 & $<0,001$ \\
\hline \multirow[t]{2}{*}{ de saúde } & Intermediária & 74 & 50,0 & & 38,1 & & 36,6 & & 67,5 & \\
\hline & Boa & 47 & 38,3 & & 61,9 & & 6,6 & & 24,3 & \\
\hline \multirow[t]{3}{*}{ Saúde comparada } & Ruim & 20 & 1,6 & & 0 & & 56,6 & & 8,1 & $<0,001$ \\
\hline & Intermediária & 51 & 33,3 & & 19,0 & & 36,6 & & 67,5 & \\
\hline & Boa & 77 & 65,0 & & 81,0 & & 6,6 & & 24,3 & \\
\hline \multirow[t]{2}{*}{ Perda de peso } & $\operatorname{Sim}$ & 65 & 35,5 & & 40,0 & & 66,7 & & 51,5 & 0,048 \\
\hline & Não & 76 & 64,5 & & 60,0 & & 33,4 & & 48,5 & \\
\hline \multirow[t]{2}{*}{ Fadiga } & $\operatorname{Sim}$ & 107 & 11,6 & & 33,4 & & 60,0 & & 22,2 & $<0,001$ \\
\hline & Não & 40 & 88,4 & & 66,6 & & 40,0 & & 77,8 & \\
\hline \multirow{2}{*}{$\begin{array}{l}\text { Baixa força de } \\
\text { preensão }\end{array}$} & $\operatorname{Sim}$ & 62 & 66,1 & & 76,2 & & 66,7 & & 29,8 & $<0,001$ \\
\hline & Não & 88 & 33,9 & & 23,8 & & 33,3 & & 70,2 & \\
\hline \multirow[t]{2}{*}{ Lentidão da marcha } & $\operatorname{Sim}$ & 68 & 56,9 & & 19,0 & & 86,7 & & 40,5 & $<0,001$ \\
\hline & Não & 82 & 40,3 & & 81,0 & & 13,3 & & 59,5 & \\
\hline \multirow[t]{2}{*}{ Inatividade física } & $\operatorname{Sim}$ & 120 & 100,0 & & 38,1 & & 80,0 & & 73,0 & $<0,001$ \\
\hline & Não & 29 & 0 & & 61,9 & & 20,0 & & 27,0 & \\
\hline \multirow[t]{2}{*}{ Conglomerados } & & & 1 & & 2 & & 3 & & 4 & \\
\hline & & $\mathrm{n}$ & $\mathrm{m}(\mathrm{dp})$ & $\mathrm{n}$ & m (dp) & $\mathrm{n}$ & $\mathrm{m}(\mathrm{dp})$ & $\mathrm{n}$ & $\mathrm{m}(\mathrm{dp})$ & valor-p ${ }^{* *}$ \\
\hline \multirow[t]{5}{*}{ AAVD } & Físicas & 61 & $1,4(0,9)$ & 21 & $\mathbf{3 , 5}(0,8)$ & 30 & $1,1(1,0)$ & 37 & $2,4(1,0)$ & $<0,001$ \\
\hline & Sociais & 61 & $3,5(1,3)$ & 21 & $5,3(1,3)$ & 30 & $2,2(1,3)$ & 37 & $3,8(1,4)$ & $<0,001$ \\
\hline & Físico-sociais & 61 & $0,1(0,3)$ & 21 & $0,2(0,4)$ & 30 & $0,1(0,2)$ & 37 & $\mathbf{0 , 8}(0,6)$ & $<0,001$ \\
\hline & Intelectuais & 61 & $\mathbf{0 , 7}(0,7)$ & 21 & $1,0(0,8)$ & 30 & $0,2(0,4)$ & 37 & $0,5(0,7)$ & $=0,001$ \\
\hline & Intel.sociais & 61 & $1,0(0,0)$ & 21 & $\mathbf{0 , 8}(0,3)$ & 30 & $0,7(0,4)$ & 37 & $1,0(0,0)$ & $<0,001$ \\
\hline
\end{tabular}

$\mathrm{R}^{2}$ total= 0,227; $\mathrm{R}^{2}$ comparação social $=0,469 ; \mathrm{R}^{2}$ AAVD físicas $=0,418 ; \mathrm{R}^{2}$ AAVD físico-sociais $=0,400 ; \mathrm{R}^{2}$ AAVD sociais $=0,302$; $R^{2}$ Inat.fís. $=0,269$.

*Teste qui-quadrado; diferença significativa se $\mathrm{p} \leq 0,05$; ${ }^{* *}$ Teste Kruskal-Wallis; diferença significativa se $\mathrm{p} \leq 0,05$. 


\section{DISCUSSÃO}

As mulheres constituíram maioria, como acontece em grande parte dos estudos populacionais com idosos da comunidade, residentes em instituições e atendidos em ambulatórios. A maior longevidade feminina foi identificada por diversos estudos, e este fato aponta para o advento da feminização da velhice, ${ }^{28} \mathrm{o}$ qual se relaciona à maior expectativa de vida da mulher em relação ao homem, resultante, entre outros fatores, das diferenças entre os gêneros quanto ao estilo de vida e às atitudes frente às doenças e incapacidades. ${ }^{1,29}$

O grupo etário predominante foi o de 70 a 79 anos. Segundo evidências da literatura, verificase um maior contingente de idosos mais velhos em serviços de referência na área de geriatria, em função do crescimento acelerado deste grupo etário ${ }^{30}$ e do baixo nível socioeconômico desse segmento entre os idosos brasileiros, típico dessa coorte.

Os idosos atendidos em ambulatório diferenciam-se daqueles hospitalizados ou residentes em instituições de longa permanência para idosos, cuja saúde e funcionalidade são geralmente mais comprometidas. Idosos que buscam assistência em ambulatórios de um hospital da rede de atenção básica à saúde são geralmente mais velhos e de baixa renda, e apresentam condições graves ou múltiplas morbidades advindas de implicações multifatoriais. Diferem de idosos ativos residentes na comunidade, que via de regra apresentam baixas taxas de fragilidade. É provável que o fato de os idosos terem sido recrutados em ambulatório de geriatria tenha determinado a alta prevalência de fragilidade encontrada.

Nesta pesquisa, observou-se que os idosos com piores condições objetivas de saúde e os frágeis apresentaram piores autoavaliações de saúde e de saúde comparada a de outros da mesma idade. Tiveram pior desempenho de AAVD, com destaque para as mulheres e para os com 80 anos e mais. Por serem mais velhos e terem maiores comprometimentos na saúde física, tendem a utilizar mais mecanismos de comparação social descendente, com a finalidade de obter alento, aumentar a autoestima com relação à saúde, proteger o self, regular emoções e manter o bemestar subjetivo. ${ }^{5}$

Woods et al. ${ }^{31}$ encontraram resultados semelhantes no Women's Health Initiative Observational Study. Borim et al. ${ }^{2}$ observaram que autoavaliação negativa de saúde associa-se com baixo status de saúde e de capacidade funcional. Rosa et al. ${ }^{12}$ identificaram que idosos com pior saúde percebida em comparação com outros da mesma idade tinham também pior capacidade funcional. Idosos que avaliam negativamente a própria saúde tendem a investir menos em autocuidado, a não praticar exercícios físicos, a ir menos ao médico e a descuidar da alimentação saudável e do desenvolvimento psicossocial. Ficam mais expostos aos riscos de desenvolver fragilidade e de pouco envolvimento social. A saúde percebida como boa ou excelente reflete uma disposição otimista geral, que indica influências protetoras em relação aos sistemas neurológico, imunológico e endocrinológico. ${ }^{32} \mathrm{~A}$ saúde percebida como pobre ou ruim é preditora de declínio funcional e de mortalidade. ${ }^{2,8}$

As AAVDs mais desempenhadas foram as do domínio social, sem diferença entre homens e mulheres, possivelmente porque parte das atividades avaliadas não contemplam esforço físico mais intenso. Os idosos que recebiam visitas em casa eram em número maior do que os que faziam visitas, uma atividade mais exigente do ponto de vista da independência física. Algumas atividades não foram citadas por grande parte dos integrantes desta pesquisa, dentre as quais "fazer trabalho remunerado e trabalho voluntário fora de casa", "participar de diretorias ou conselhos e de cursos ou grupos de estudo", práticas que envolvem compromissos diários ou semanais, motivação e repertório prévio, de 
baixa probabilidade de ocorrência entre os muito idosos, com doenças crônicas e com problemas de mobilidade. Grande parte relatou costumar ficar em casa assistindo televisão, optando pelo lazer passivo.

No médio prazo, o abandono de AAVD provoca perdas em sociabilidade e em bem-estar subjetivo. Nunes et al. ${ }^{8}$ destacam a importância do engajamento social para o envelhecimento ativo. Para muitos idosos, faltam oportunidades para o desenvolvimento de um repertório mais diversificado de atividades.

A inexistência de locais apropriados nas imediações do domicílio, as dificuldades financeiras ou mesmo físicas e cognitivas podem ser causas da baixa frequência dos idosos em atividades sociais. Além disso, este estudo não questionou a preferência pregressa do idoso para as atividades, restando dúvidas sobre as razões pelas quais não praticavam parte delas - se por incapacidade, desinteresse ou falta de oportunidade.

No domínio físico, configurou-se a maior diferença entre os gêneros. Os homens têm maior tradição de prática de exercícios físicos recreativos no decorrer da vida e são menos acometidos por enfermidades que causam dor, como as artrites, do que as mulheres. Além disso, fatores históricos e culturais que determinam que as mulheres fiquem mais em casa e assim pratiquem menos atividades físicas também precisam ser considerados.

Constatou-se que entre os idosos com desempenho mais fraco em AAVD havia maior número de frágeis, com destaque para as mulheres e para os mais velhos. Existem preconceitos e atitudes negativas com relação à velhice que, associadas à ideia de perdas em AAVD como normativas, impedem que medidas de intervenção sejam tomadas. Tais preconceitos podem associar-se à diminuição da confiança dos idosos em suas capacidades, modificar a percepção da própria saúde e diminuir o envolvimento ativo com a vida, resultando em isolamento social e em prejuízos gerais para a qualidade de vida. Essas ocorrências são particularmente prejudiciais ao bem-estar dos idosos assistidos em ambiente ambulatorial.

Por outro lado, a participação em AAVD é influenciada por condições sociais, educacionais, financeiras, ambientais e de saúde. Dados de estudos longitudinais mostram que as mulheres são socialmente mais ativas, participativas e envolvidas, se comparadas aos homens, e que limitações em saúde e funcionalidade afastam mais homens do que mulheres das atividades sociais, ${ }^{33}$ sugerindo a atuação de variáveis motivacionais e culturais associadas a gênero. ${ }^{34}$ Segundo Scheibe \& Carstensen, ${ }^{35} \mathrm{o}$ afastamento de atividades e de papéis sociais pode ser decorrência das prioridades socioemocionais dos idosos, que tendem a substituir atividades e metas associadas à busca de informação e de status por outras que trazem conforto emocional. Assim, os idosos tendem a privilegiar atividades sociais no ambiente mais próximo, ocupações estas compatíveis com suas condições físicas, educacionais e motivacionais. ${ }^{34}$

Em síntese, os idosos com piores condições objetivas de saúde e os frágeis apresentaram piores autoavaliações de saúde e de saúde comparada à de outros da mesma idade e tiveram baixo desempenho em AAVD. Este resultado expressa as características da amostra investigada, uma vez que os idosos ambulatoriais, em sua maioria frágeis, são pouco ativos socialmente. Este fato sugere a influência não só das condições físicas adversas, como também de comprometimento motivacional e emocional.

\section{CONCLUSÕES}

A saúde é uma preocupação central na vida dos idosos. Pelos custos financeiros, pessoais e familiares acarretados por doenças crônicas e incapacidades, é também uma preocupação para os governos e para os sistemas públicos e privados de saúde e previdência social. No âmbito individual, o que os idosos pensam e dizem a respeito da própria saúde desempenha papel orientador em relação ao autoconceito, à motivação para atividades, ao autocuidado e à adesão a tratamentos preconizados por profissionais. 
Este estudo investigou a relação entre saúde subjetiva, envolvimento em atividade social e fragilidade no envelhecimento. A autoavaliação de saúde positiva e a manutenção do envolvimento social integram condições para amenizar o impacto da possível diminuição do desempenho de atividades complexas de vida diária em idosos frágeis ambulatoriais, o que torna importante a investigação dessas relações.

\section{REFERÊNCIAS}

1. Lima-Costa MF, Matos DL, Camargos VP, Macinko J. 10-year trends in the health of Brazilian elderly: evidence from the National Household Sample Survey (PNAD 1998, 2003, 2008). Ciênc Saúde Coletiva 2011;16(9):3689-96.

2. Borim FSA, Barros MBA, Neri, AL. Autoavaliação de saúde em idosos: pesquisa de base populacional no município de Campinas, São Paulo, Brasil. Cad Saúde Pública 2012;28(4):769-80.

3. Ware JE Jr. Scales for Measuring General Health Perceptions. Health Serv Res 1976;11(4):396-415.

4. Festinger L. A theory of social comparison processes. Hum Relat 1954;7:117-40.

5. Neri AL, organizadora. Qualidade de vida na velhice: enfoque multidisciplinar. Campinas: Alínea; 2007. p.13-59.

6. Lima-Costa MF, Firmo JOA, Uchôa E. A estrutura da auto-avaliação da saúde entre idosos: projeto Bambuí. Rev Saúde Pública 2004;38(6):827-34.

7. Lima-Costa MF, Firmo JOA, Uchôa E. Diferenças na estrutura da auto-avaliação da saúde em idosos com diferente situação sócio-econômica: Projeto Bambuí. Cad Saúde Pública 2005;21(3):830-39.

8. Nunes APN, Barreto SM, Gonçalves LG. Social relations and self- rated health: the ageing and health project. Rev Bras Epidemiol 2012;15(2):415-28.

9. Blazer DG. How Do You Feel About. . .? Health outcomes in late life and self-perceptions of health and well-being. Gerontologist 2008;48(4):415-22.

10. Guariento ME, White HJ, Todoni MI, Rodrigues NO. Acesso, uso e satisfação em relação aos serviços de saúde e autoavaliação do autocuidado com a saúde. In: Neri AL, Guariento ME, organizadoras. Fragilidade, saúde e bem-estar em idosos: dados do estudo FIBRA Campinas. Campinas: Alínea, 2011. p. 149-70.

11. Rodrigues MAP, Facchini LA, Piccini RX, Tomasi E, Thumé E, Silveira DS, et al . Use of outpatient services by the elderly in the South and Northeast of Brazil. Cad Saúde Pública 2008;24(10):2267-78.

12. Rosa TEC, Benício MHD, Latorre MRDO, Ramos LR. Fatores determinantes da capacidade funcional entre idosos. Rev Saúde Pública 2003;37(1):40-8.

13. Luz TCB, César CC, Lima-Costa MF, Proietti FA. Satisfaction with the neighborhood environment and health in older elderly: cross-sectional evidence from the Bambuí Cohort Study of Aging. Cad Saúde Pública 2011;27 Sup 3:S390-8.

14. Cornwell EY, Waite LJ. Social disconnectedness, perceived isolation, and health among older adults. J Health Soc Behav 2009;50(1):31-48.

15. Organização Panamericana de Saúde, Organização Mundial da Saúde. Classificação Internacional de funcionalidade, incapacidade e saúde (CIF). São Paulo:Edusp; 2011.

16. Reuben DB, Laliberte L, Hiris J, Mor V. A hierarchical exercise scale to measure function at the advanced activities of daily living (AADL) level. J Am Geriatr Soc 1990;38(8):855-61.

17. Baltes MM, Mayr U, Borchelt M, Mass I, Wilms HU. Everday competence in old and very old age: an interdisciplinary perspective. Ageing Soc 1993;13:657-80.

18. Vermeulen J, Neyens JCL, Van Rossum E, Spreeuwenberg MD, De Witte LP. Predicting ADL disability in community-dwelling elderly people using physical frailty indicators: a systematic review. BMC Geriatr 2011;11:1-11.

19. Al Snih S, Graham JE, Ray LA, Samper-Ternent R, Makjdes KS, Ottenbarcher KJ. Frailty and incidence of activities of daily living disability among older Mexican Americans. J Rehabil Med 2009;41(11):892-7.

20. Kono A, Kai I, Sakato C, Rubenstein LZ. Frequency of going outdoors predicts long-range functional change among ambulatory frail elders living at home. Arch Gerontol Geriatr 2007;45(3):233-42.

21. Linhares CRC, Coelho VLD, Guimarães, RM, Campos APM, Carvalho NT. Perfil da Clientela de um Ambulatório de Geriatria do Distrito Federal. Psicol Reflex Crít 2003;16(2):319-26. 
22. Fried LP, Tangen CM, Walston J, Newman AB, Hirsch C, Gottdiener J, et al. Frailty in older adults: evidence for a phenotype. J Gerontol Ser A Biol Sci Med Sci 2001;56(3): M146-56.

23. Yesavage JA, Brink TL, Rose TL, Lum O, Huang $\mathrm{V}$, Adey M, et al. Development and validation of a geriatric depression screening scale: a preliminary report. J Psychiatr Res 1983;17(1):37-49.

24. Batistoni SST, Neri AL, Cupertino APFB. Validity of the Center for Epidemiological Studies Depression Scale among Brazilian elderly. Rev Saúde Pública 2007;41(4):598-605.

25. Haskell WL, Lee IM, Pate RR, Powell KE, Blair SN, Franklin BA, et al. Physical activity and public health: updated recommendation for adults from the American College of Sports Medicine and the American Heart Association. Med Sci Sports Exerc 2007;39:1423-34.

26. Nelson ME, Rejeski WJ, Blair SN, Duncan PW, Juiz JO, Rei AC, et al. Physical activity and public health in older adults: recommendation from the American College of Sports Medicine and the American Heart Association. Circulation 2007;116:1094-1105.

27. American College of Sports Medicine; ChodzkoZajko WJ, Proctor DN, Fiatarone SMA, Minson CT, Nigg CR, Salem GJ, et al. American College of Sports Medicine position stand. Exercise and physical activity for older adults. Med Sci Sports Exerc. 2009;41(7):1510-30.
28. Camarano AA, Pasinato MT, Lemos VR. Cuidados de longa duração para a população idosa. Uma questão de gênero? In: Neri AL, organizadora. Qualidade de Vida na Velhice: enfoque multidisciplinar. Campinas: Alínea; 2007. p.127-49.

29. Louvison MC, Lebrão ML, Duarte YAO, Santos JLF, Malik AM, Almeida ES. Inequalities in access to health care services and utilization for the elderly in Sao Paulo, Brazil. Rev Saúde Pública 2008;42(4):733-40.

30. Rosset I, Pedrazzi EC, Roriz-Cruz M, De Morais EP, Rodrigues RAP. Tendências dos estudos com idosos mais velhos na comunidade: uma revisão sistemática internacional. Rev Esc Enferm USP 2011;45(1):264-71.

31. Woods NF, LaCroix AZ, Gray SL, Aragaki A, Cochrane BB, Brunner RL, et al. Frailty: Emergence and consequences in Women Aged 65 and older in the Women's Health Initiative Observational Study. J Am Geriatr Soc 2005;53(8):1321-30.

32. Lyyra, TM, Heikkinen E, Lyyra AL, Jylhä M. Self-rated health and mortality: Could clinical and performance-based measures of health and functioning explain the association? Arch Gerontol Geriatr 2006;42(3):277-88.

33. Thomas PA. Gender, social engagement, and limitations in late life. Soc Sci Med 2011;73(9):1428-35.

34. Neri AL, organizadora. Fragilidade e qualidade de vida na velhice. Campinas: Alínea; 2012. p. 247-66.

35. Scheibe S, Carstensen LL. Emotional aging: recent findings and future trends. J Gerontol B Psychol Sci Soc Sci 2010;65(2):135-44. 